\title{
El movimiento 15-m en los medios y en las redes. Un análisis de sus estrategias comunicativas
}

\author{
The 15-m movement in the media and in networks: an analysis \\ of communication strategies
}

\author{
José Manuel Robles, Antón R. Castromil, Arturo Rodríguez y \\ Mildred Cruz \\ Universidad Complutense de Madrid \\ jmrobles@ccee.ucm.es(ESPAÑA)
}

RubÉn DíEz

Universidad Carlos III

Recibido: 19.062015

Aceptado: 22 .09.2015

\section{RESUMEN}

Este trabajo se centra en el análisis de los usos de Internet por parte de los activistas del movimiento 15-M en dos sentidos: i) como vehículo de comunicación con sus potenciales audiencias y ii) como estrategia que permite poner en circulación marcos alternativos a los ofrecidos por los mass media. Por ello analizamos, también, el papel que jugó la prensa escrita durante las elecciones autonómicas y municipales de 2011 cuando el movimiento irrumpió con fuerza en la vida pública española. Frente a los marcos desarrollados por la prensa escrita durante dicho periodo, los activistas del movimiento reaccionaron con una estrategia dirigida a controlar el flujo de información sobre lo que estaba sucediendo en las plazas de muchas ciudades de España. Se trata, de una estrategia de acción comunicativa que gira en torno al marco general del 15-M y que, gracias en gran medida a Internet, se centra en la necesidad de desarrollar formas de participación directas y des-intermediar las relaciones entre los activistas y el resto de ciudadanos. 


\title{
PALABRAS CLAVE
}

Internet, Participación política digital, 15-M y mass media

\begin{abstract}
This paper focuses on the analysis of the uses of the Internet by activists of the 15-M movement. We pay attention to two processes: the use of the Internet i) as a vehicle of communication with their potential audiences and ii) as a technology that allows to circulate an alternative framework. Therefore we analyzed also the role played by the newspapers during the regional and municipal elections of 2011 when the movement burst onto the Spanish public life. Facing the frameworks developed by the newspapers during this period, the activists reacted with a strategy to control the flow of information about what was happening in the streets of many cities in Spain. This strategy of communicative action revolves around the general framework of the 15-M and, thanks largely to the Internet, is focused on the need to develop forms of direct participation and the disintermediation of the relationships between activists and other citizens.
\end{abstract}

\section{KEY WORDS}

Internet, digital political participation, 15-M, mass media.

\section{INTRODUCCIÓN}

El estudio de los Movimientos Sociales se encuentra, desde hace algunos años, estrechamente vinculado al papel que juega Internet como herramienta para la coordinación de la acción colectiva, como vehículo para el reclutamiento de nuevos simpatizantes, así como espacio en el que se amplifican las acciones de protesta (Bennet y Segerberg, 2013). Igualmente, uno de los ámbitos más relevantes y prolíficos en el estudio de la relación entre Movimientos Sociales e Internet está relacionado con la capacidad de este tipo de organizaciones para gestionar la información y pugnar en el espacio público con las instituciones que, tradicionalmente, copaban dicho espacio. En este terreno, una de las preguntas más relevantes se plantea en qué medida Internet ha posibilitado o no una mayor independencia informativa de los movimientos sociales y hasta qué punto ha potenciado el papel de estas organizaciones como creadoras de contenidos culturales (Castells, 2012).

Nuestro trabajo se centra en este ámbito de estudio para analizar el uso político de Internet por parte del movimiento 15-M (Robles y Ganuza, 2011) y, en concreto, 1) cómo percibían sus activistas las posibilidades abiertas por Internet como vehículo de comunicación con sus potenciales audiencias, 2) qué papel jugó la prensa escrita durante el proceso de mayor actividad del 
movimiento 15-M y 3) cómo dicho movimiento desarrolló una estrategia para, gracias a Internet y en especial a las redes sociales, poner en circulación información alternativa a la ofrecida por mass media. Nuestra hipótesis general es que Internet, al reducir los costes de gestión y distribución de la información, permite al 15-M evitar la mediación que, tradicionalmente, llevan a cabo los medios. Sin embargo, evitar la mediación no supone negar dichos canales de comunicación que, como veremos, son en muchos casos los principales medios de información política para amplias capas de la ciudadanía. Tampoco implica transformar Internet en el único medio a través del cual los activistas presentan sus ideas y reivindicaciones. Des-intermediar significa aquí tratar de disminuir la probabilidad de que la información sobre el movimiento sea elaborada, basándose en los valores e intereses, de otros colectivos (periodistas, líderes de opinión, etc.) que no sean el propio movimiento. Evitar la mediación implica controlar la información que se emite haciendo de las redes sociales digitales del 15-M una fuente directa de información. En este artículo veremos, igualmente, cómo esta estrategia enlaza con el marco general del movimiento según el cual la forma apropiada de regeneración política pasa por una intervención más directa y des-intermediada de los ciudadanos en las distintas esferas del poder.

Para alcanzar este objetivo, procederemos de la siguiente forma. En primer lugar, planteamos una reconstrucción del proceso de desarrollo del 15-M atendiendo, principalmente, a la relación entre dicho movimiento y los distintos medios de comunicación. En segundo lugar, proponemos un breve marco teórico en el que se especifica el sentido de los conceptos e ideas que enmarcan nuestro trabajo. En tercer lugar, planteamos un estudio empírico en dos partes. La primera, estudia cómo enmarca la prensa nacional el fenómeno 15-M y, la segunda, analiza la reacción de los activistas del movimiento, así como su estrategia explícita para evitar el control mediático del flujo de información sobre el movimiento. Por último, ofrecemos una serie de conclusiones que nos permiten sintetizar la información ofrecida en el trabajo y reflexionar sobre el sentido y profundidad de las hipótesis aquí planteadas.

\section{BREVE INTRODUCCIÓN AL MOVIMIENTO 15-M, LOS NEW MEDIA \& LOS MASS MEDIA}

Cuatro años después de la emergencia del movimiento 15-M, y su significativa y continuada visibilidad pública en las calles desde el periodo de las elecciones municipales de Mayo de 2011, pocos autores cuestionan las repercusiones y los logros de este movimiento. El 15-M se ha fundamentado sobre una ciudadanía indignada que ha sintonizado con sus marcos de acción colectiva apoyándolo en sus convocatorias públicas y despertando grandes simpatías en el plano simbólico (Laraña \& Díez, 2012; Antón, 2012; Sampedro \& Lobera, 2014; Díez, 2015). Este movimiento ha vigorizado a la sociedad civil española, que ya se venía presentado muy activa y abierta desde mediados de los años noventa del siglo pasado (Laraña, 2007, 2009; Laraña y Díez, 2010; 2012; 
2013), y lo ha hecho en diferentes órdenes y planos de la agenda y de la vida pública y privada de numerosos ciudadanos. Apoyándose y valiéndose de una amplia variedad de actividades y acciones con un alto grado de reflexividad, que lo han conformado como una agencia de significación y persuasión colectiva, el movimiento 15-M ha resonado con fuerza en la vida social y cultural de España y en el plano de la política institucional, así como en el ámbito internacional.

A lo largo de este proceso, que abarca desde 2011 a 2015, tanto las tecnologías digitales de la comunicación, como los mass media han jugado un papel clave en el desarrollo y las transformaciones del movimiento. Desde su misma génesis, aún con un alto grado de latencia en la vida pública y los medios de comunicación tradicionales, pero no en la web, las tecnologías digitales de la comunicación fueron fundamentales en las fases previas a la movilización del 15 de mayo de 2011 y durante las acampadas que le dan nombre. Y con las que se le identifica con frecuencia entre la opinión pública y los medios de comunicación tradicionales. Por otro lado, desde sus inicios el movimiento de los indignados presentó un alto grado de pluralismo y un carácter transversal (Laraña y Díez, 2012), que contribuyó al alineamiento de los marcos de las organizaciones y colectivos de este movimiento con los valores y demandas de la ciudadanía indignada. Razón por la cual ha sido caracterizado, también, como un movimiento de 'ciudadanos' o 'personas', (Fernández-Savater, 2011; 2012; 2014; Perugorría y Tejerina, 2013:10), mientras que otros autores y perspectivas ponen el acento en sus continuidades con experiencias activistas y militantes particulares previas (Maeckelbergh, 2012; Fominaya, 2014). Este carácter transversal, su pluralismo ideológico y la significativa variedad de tradiciones y prácticas activistas que ha aglutinado en su seno, parecen haber sido determinantes en el proceso de construcción (y transformación) de su identidad colectiva, fruto de las dinámicas de interacción, negociación y conflicto que se han generado en las redes del, y en torno al, movimiento y fuera de él y en la ampliación y la extensión de sus marcos de acción colectiva (Díez, 2015)․․

Uno de los rasgos substanciales que está en la base de su marco de acción colectiva reside en su fuerte crítica a los grandes partidos políticos tradicionales que vincula con su reivindicación de una mayor democracia y su demanda de cambio social. En estas reivindicaciones se reserva, asimismo, un papel clave a las tecnologías digitales como nuevas herramientas democráticas. Una democracia que se ve amenazada por la connivencia entre la clase política y poderosos grupos plutocráticos, aspecto que da lugar a una atribución de identidades y a un marco de diagnóstico ampliado por parte del movimiento, que define a las organizaciones políticas tradicionales y grupos plutocráticos como los responsables de la falta de soluciones a los problemas cotidianos de la

${ }^{1}$ Las divergencias que parecen aparecer en la caracterización de este movimiento cobran sentido si entendemos que los movimientos sociales están sujetos a un proceso de construcción de su identidad colectiva (Melucci, 1989, 1996). Según las lentes, los espacios, los repertorios, los marcos o el momento en el que el analista pone el foco a lo largo de estos cuatro años, la caracterización de dicha identidad puede variar. 
ciudadanía y de los graves problemas económicos que ha enfrentado España y otros países del sur de Europa desde 2008 (Laraña, y Díez, 2012).

La emergencia del 15-M ha puesto de manifiesto una tendencia que ya venía siendo observable desde hace tiempo. Las tecnologías digitales de la comunicación juegan hoy en día un papel de primer orden en la participación de los ciudadanos en ámbitos menos visibles y alejados de la política formal. En la expansión de nuevas formas políticas, 冈la subpolítica (Beck, 1992) 囚, que implican la apertura de nuevos cauces y esferas de participación. El uso de Internet y de aplicaciones con un alto nivel de penetración entre la población como Facebook o Twitter favorecen este hecho, así como la difusión y la resonancia de los mensajes y demandas de las organizaciones de la sociedad civil y de los movimientos sociales. Así por ejemplo, autores como Castells (2012:2) enfatizan el papel determinante de las tecnologías digitales de la comunicación y de su estructura en red en la difusión rápida y viral de nuevas ideas y mensajes que favorecen la extensión y el contagio de los movimientos sociales en el ámbito nacional y en la esfera internacional.

Asimismo, los medios de comunicación tradicionales han seguido jugando un papel clave en las dinámicas de movilización social, esto es, en la difusión y resonancia de sus marcos de la acción colectiva y en la construcción de su identidad pública y colectiva (Johnston, Laraña y Gusfield, 1994). Debido a la importancia de los medios de comunicación de masas en las actividades de significación colectiva, los movimientos sociales con un mayor poder de convocatoria en sus acciones públicas son depositarios de una mayor capacidad de influencia en el terreno simbólico, ya que dicho poder de convocatoria los convierte en noticia e incrementa su potencial como agencias de persuasión colectiva (Laraña, 1999).

En este trabajo encuadramos la génesis y el desarrollo del movimiento 15-M en este triple marco: i) en el de nuevas formas de acción política en ámbitos menos visibles y alejados de la política institucional, ii) en el inteligente uso de las tecnologías digitales de la comunicación y su estructura descentralizada y en red, dinamizada por prácticas sociales y modos de interacción colaborativos (Benkler, 2006), y iii) en el de su cobertura por parte de los medios de comunicación tradicionales.

La propia génesis del movimiento está asociada al "movimiento por una cultura libre' y al uso de las redes sociales digitales y a la red de redes (Castells, 2012; Toret, 2012; Candón, 2013) ${ }^{2}$. Y no se puede entender, al margen de numerosísimos otros factores, sin estas aplicaciones tecnológicas de amplia penetración. Por ejemplo, la creación de un grupo de Facebook en 2011, la "Plataforma de Coordinación de Grupos por la Movilización Ciudadana", y

2 Uno de los grupos más activos fue precisamente 'No les Votes', muy activos y críticos en Internet con los partidos políticos que, como el PSOE, el PP o CIU, apoyaron la 'Ley Sinde'. Esta normativa introducía modificaciones legales y nuevas regulaciones en la Ley de Servicios de la Sociedad de la Información y en la Ley de Propiedad Intelectual en el ámbito de los citados servicios de la sociedad de la información y el comercio electrónico. 
de numerosas cuentas de Twitter y otros canales (v.g., meneame.net), como modos de organización y señas de identidad de los diferentes colectivos y organizaciones que conformaron la Plataforma 'Democracia Real Ya!' . A dicho grupo de Facebook se fueron uniendo "espontáneos, blogueros, gente del Movimiento Ciudadano Estado del Malestar, la plataforma 'No les votes", (Elola, 2011) y finalmente evolucionó en un foro de debate y acción en esta red social que fue denominado 'Democracia Real Ya!'. A esta Plataforma se sumaron organizaciones como Juventud Sin Futuro o la Plataforma de Afectados por la Hipoteca, y convocaron una manifestación coordinada y organizada a través de las redes sociales en Internet y en redes de interacción cara a cara en reuniones y asambleas que, en Madrid, tuvieron lugar en el Patio Maravillas. Estas redes latentes ${ }^{4}$ se visibilizaron en la manifestación del 15 de Mayo de 2011 en Madrid, que desencadenó posteriormente en las acampadas de los indignados de la Puerta del Sol tras el primer intento de desalojo por parte de la policía de algunas de las personas que habían participado en la manifestación.

El significado que el movimiento 15-M ha atribuido a las tecnologías digitales de la comunicación y su relación con los mass media, como hemos aludido con anterioridad, son elementos clave en la construcción de su identidad pública y colectiva, y en su reivindicación de una democracia más participativa. En un trabajo anterior (Laraña y Díez, 2012) enfatizaban como 'la autoafirmación de identidad que produjeron algunas definiciones colectivas de los problemas por los que se movilizaron, que fueron difundidas por los activistas a través de las redes sociales छy por lo mass media区, se reflejaba en el lema Democracia Real Ya: No somos mercancías en manos de políticos y banqueros'. Tal autoafirmación de identidad y definición de la situación que presenta a los ciudadanos como mercancías manejadas por políticos y banqueros, tuvo una gran resonancia gracias al inteligente uso de estas tecnologías y de su cobertura por los medios de comunicación tradicionales. No obstante, las tecnologías digitales de la comunicación no sólo fueron utilizadas como herramientas de difusión, también fueron empleadas

3 "Empezamos a organizarnos creando un grupo en Facebook y pronto tomamos Twitter, Youtube y Tuenti (las redes sociales más utilizadas en el Estado español) para extender el mensaje de la convocatoria, pero sobre todo para hacer fácil el paso de simpatizar con la campaña a formar parte de ella, rompiendo la frontera entre admirar un proceso e incorporarse activamente en él. Las personas que empezaban a unirse no se conocían entre sí, éramos de distintas ciudades. En apenas unos meses de trabajo en la red construimos una increíble energía cooperativa capaz de envolver a miles de personas en una campaña para la movilización del 15 de mayo" (Toret, 2012, pp. 52-53).

4 En el espacio físico, pero no tanto en el digital. No obstante, con anterioridad, el 7 de abril de 2011, Juventud Sin Futuro ya celebró una manifestación con un importante poder de convocatoria en Madrid. Asimismo, colectivos como Estado del Malestar o Juventud en Acción llevaron a cabo, con mayor o menor éxito de participación y convocatoria, algunas acciones públicas: https:// www.facebook.com/events/187971287897895 (Juventud en Acción, Madrid, 23/01/2011); https:// www.youtube.com/watch?v=rRxwHRHG8ys (Estado del Malestar, Madrid 04/03/2011); https:// www.youtube.com/watch?v=2hqz-84TDm0 (Estado del Malestar, Madrid, 'Los viernes a Sol', 18/03/2011); https://www.youtube.com/watch?v=ZVsfMOnZZS8 (Estado del Malestar, Madrid, ‘AAbrazos Gratis!', 25/03/2011).

EMPIRIA. Revista de Metodología de Ciencias Sociales. N. ${ }^{\circ}$ 32, septiembre-diciembre, 2015, pp. $37-62$. ISSN: 1139-5737, DOI/empiria. 32.2015.15308 
como medios de organización, coordinación y de movilización del consenso (Klandermans, 1984), en sus inicios y sobremanera, algunas de ellas, cuando el movimiento alcanzó mayores cotas de apoyo y expansión en el territorio a través de asambleas en barrios y municipios. Mientras que los medios de comunicación tradicionales también parecen haber desempeñado un papel clave en la capacidad del 15-M para movilizar a sus potenciales seguidores y simpatizantes.

En el caso de este movimiento, la centralidad en el uso de las aplicaciones de mayor penetración dio paso (si bien en convivencia y sin desplazarlas necesariamente) a otras más específicas, algunas de ellas, con un rango colaborativo amplio. Estas han sido herramientas de uso común para muchas de las personas más activas e implicadas en las diferentes asambleas, grupos, colectivos e iniciativas surgidas de las redes del movimiento. Por ejemplo, aplicaciones sencillas para editar textos de forma colaborativa como TitanPad, aplicaciones como Mumble, para la celebración de asambleas y videoconferencias, o plataformas como Bambuser, para streaming. Así como redes sociales alternativas con un gran potencial colaborativo como N-1 o redes sociales ad hoc como la propia de Democracia Real Ya!, —el Ágora de la Red DRY.

A un tiempo han ido surgiendo y utilizándose numerosos proyectos, iniciativas y herramientas colaborativas que ponen el acento en los procesos de deliberación y debate, y en la toma de decisiones de forma participativa y democrática. Medios tecnológicos con un gran potencial para impulsar formas más participativas de entender la política institucional y la democracia, como por ejemplo el proyecto de amplio alcance Democracia $4.0^{5}$. Hasta otras herramientas de alcance medio (v.g., Propongo, Agora Voting, Loomio, Reddit o AppGree), con un gran predicamento entre las iniciativas y organizaciones políticas que han surgido en los últimos años tras la emergencia del 15-M. Estas son nuevas organizaciones políticas que han dado el salto a la arena institucional, como por ejemplo, el Partido X, Podemos, Guanyem Barcelona/Barcelona en Comú o Ahora Madrid, la marca de confluencia para la ciudad de Madrid de Podemos y Ganemos Madrid _ — ; cuya cobertura por parte de los mass media tiene también gran interés, si bien no es el objeto central de este trabajo y queda para análisis futuros.

5 Ideada para compaginar el voto de los representantes políticos en el Congreso de los Diputados y la participación ciudadana mediante el voto de los ciudadanos a través de Internet y bajo certificado digital

6 Estas organizaciones, no derivan necesariamente y de forma automática de las redes del movimiento, sino que beben de ellas (de parte de sus seguidores, simpatizantes, discursos, formas y repertorios), y están impulsadas de forma sustancial por redes de activistas y profesionales, con experiencias políticas y militantes pre-15-M. Esto es, experiencias previas y anteriores a la irrupción de este movimiento en la vida pública, que se volcaron e involucraron en las distintas fases de su proceso de desarrollo, dado su carácter inclusivo y transversal, así como el impulso y la vigorización de la sociedad civil que este ha significado. 


\section{MARCO TEÓRICO}

\subsection{Participación política e Internet}

Dados los objetivos de este artículo, expresados en la introducción, uno de nuestros focos de atención será la participación política digital no convencional y, más concretamente, en su dimensión expresiva (Robles, 2015). Con esto nos referimos al uso de herramientas digitales, fundamentalmente redes sociales digitales, interpretadas colectivamente como espacios alternativos ${ }^{7}$ para la innovación y la experimentación en la expresión de demandas políticas. Gracias a este tipo de soportes digitales, así como a la posibilidad que estos ofrecen para modificar archivos previamente grabados o para crear nuevos con cierta facilidad, los ciudadanos pueden expresar un alto grado de creatividad. Este es el caso, por ejemplo, de los memes ${ }^{8}$ de contenido social y político a través de los cuales los ciudadanos expresan, generalmente a través del humor, una determinada posición respecto a un acontecimiento político, sobre un representante político, etc.

Igualmente, tomaremos como referente uno de los enfoques más influyentes en el estudio sobre la relación entre política e Internet. Este enfoque defiende la tesis según la cual el uso de las herramientas digitales afectan a las estructuras y procesos políticos e influyen en la naturaleza y características del sistema democrático (Chadwick, 2006; Chadwick y Howard, 2009). De esta forma, los especialistas interpretan mayoritariamente el desarrollo político de Internet como un proceso político emergente y se centran en el análisis de la forma en que este proceso influye sobre la naturaleza y características del sistema político

Esta interpretación ha motivado que las definiciones del objeto de estudio enfaticen el efecto de la participación política digital sobre la estructura política de una determinada comunidad. Así, Macintosh (2006) define participación política digital como «el uso de las tecnologías de la información y la comunicación para ampliar la participación política de los ciudadanos facilitando la interconexión entre ellos, así como con sus representantes políticos» (Macintosh, 2006:123). En esta misma línea, se ha definido democracia digital como la unión entre, por una parte, la estructura democrática de una comunidad política y el ejercicio de sus funciones de gobierno y, por otra, el uso de las TIC, fundamentalmente Internet (Van Dijk, 2000)

Encontramos en esta literatura distintas interpretaciones sobre la forma que adopta esta relación. Ward y Gibson (2009) se refieren a ellas como normalización, igualación y desintermediación. Según la teoría de la normalización las relaciones sociopolíticas existentes en el ámbito off-line se están trasladando al ámbito político on-line y no encontramos, por lo

7 Utilizamos aquí "alternativos" para referirnos, tal y como quedará de manifiesto en este artículo, al uso político de las redes sociales como alternativa a los canales controlados por empresas y partidos políticos.

8 Con "memes" nos referimos a fotos y/o montajes fotográficos que han sido creados o modificados para expresar mensajes o ideas.

EMPIRIA. Revista de Metodología de Ciencias Sociales. N. ${ }^{\circ} 32$, septiembre-diciembre, 2015, pp. $37-62$. ISSN: 1139-5737, DOI/empiria. 32.2015.15308 
tanto, un efecto innovador de Internet sobre la política. Frente a esta postura, algunos estudios han mostrado que, aunque modesto, Internet sí tiene un efecto sobre las relaciones entre organizaciones políticas y entre estas y los propios ciudadanos (Bimber, 2001). Desde este punto de vista, Internet favorece un escenario políticamente más plural al permitir a las organizaciones políticas más débiles alcanzar audiencias que, aun no pudiendo competir con las que alcanzan las organizaciones políticas más poderosas, serían difíciles de lograr sin la existencia de este medio (Bennett, 2003). Por último, la tesis de la desintermedicación plantea que Internet permite relativizar el peso social y político de las organizaciones que tradicionalmente atesoraban mayor poder (Wring y Horrocks, 2001). Así, Internet se está transformando en una herramienta, gracias a la cual, los ciudadanos pueden relacionarse, gestionar y transmitir información y conocimiento sin la mediación de las instituciones características del espacio público tradicional como los medios, las organizaciones civiles, los partidos políticos, los sindicatos, etc. (Wring y Horrocks, 2001). Como veremos más adelante, este último enfoque es, desde nuestro punto de vista, el más útil para entender el proceso que tratamos de describir en este trabajo.

Este enfoque es también el mayoritario en el estudio del uso de redes sociales digitales para la participación política. Así, distintos autores se han centrado en el efecto de redes digitales concretas como Twitter o Facebook sobre las campañas electorales en distintos países del mundo (Williams y Gulati, 2013), las revueltas políticas o los procesos de acción colectiva (Bennett y Segerbert, 2013; Dylko et al., 2012). Estos estudios, en mayor o menor medida, están dirigidos a medir el efecto de distintas prácticas realizadas a través de las redes sociales digitales sobre la relación entre ciudadanos o, entre estos, y sus representantes (Dalton, 2010). En definitiva, tratan de medir el efecto del uso político de las redes sociales digitales sobre la estructura política.

\subsection{Los medios y su reflejo de la participación política}

Como hemos visto previamente, el presente trabajo aborda el análisis del movimiento 15-M y, en concreto, el efecto de Internet sobre sus posibilidades comunicativas. Sin embargo, hemos considerado oportuno analizar, como contra-parte, la construcción de la imagen pública que hicieron los medios de comunicación más tradicionales respecto al movimiento. Consideramos que, precisamente el intento de evitar la interpretación del movimiento a partir los esquemas mediáticos basados en la polaridad derecha-izquierda o PP-PSOE, está en la base de la estrategia de comunicación mediática del 15-M.

La primera cuestión destacable en este proceso es que según los datos de encuesta disponibles ${ }^{9}$, el tipo de herramientas comunicativas utilizadas extensivamente por el movimiento de los "indignados" -blogs, foros, redes sociales- no han sido las mismas que han empleado la mayoría de la población

${ }^{9}$ CIS, encuesta post electoral generales 2011 (estudio 2920) 
española a la hora de proveerse de información política. La televisión, la prensa escrita y la radio siguen siendo los modos preferidos por los ciudadanos para informarse del transcurso de la campaña. Al menos en 2011.

Esta circunstancia redunda en la necesidad de analizar los medios tradicionales para conocer la construcción pública (encuadre) que estos mismos medios trasladan a la ciudadanía. Pero, como todo proceso mediatizado, tropieza o, más bien, tiene que lidiar con la acción no inocente de los periodistas y las empresas en las que trabajan.

Para entender la imagen y el tratamiento que recibió el 15-M por parte de la prensa haremos un análisis a tres niveles a) el proceso de construcción de la agenda mediática (agenda setting), b) el encuadre de tales temas (framing) y, finalmente, c) el impacto de la polarización político-mediática del sistema de medios español en el tratamiento del 15-M.

Los datos de seguimiento de la cobertura que la prensa de referencia $(E l$ País, El Mundo, ABC y Público ${ }^{10}$ realizó de la campaña electoral de mayo de 2011 demuestran que el 15-M entró a formar parte de "núcleo duro" la campaña. Según el estudio clásico de McCombs y Shaw (1972) tal inclusión implica que los ciudadanos conocerán el tema y, a través de la discusión sobre esa issue, se irá formando poco a poco la opinión pública en torno a esa cuestión.

Esta es la principal función que los medios de comunicación tienen en todo sistema democrático: reorientar el debate en torno a un único puñado de grandes temas de debate (Ibíd.). Tal y como se demostrará a lo largo de este trabajo el 15-M será uno de estas cuestiones de debate público que los medios seleccionan en el seno de la campaña electoral.

Pero los medios de comunicación no se limitan sólo a la selección de temas, ofrecen una determinada perspectiva (Entman, 1993; McCombs et al 1998) dentro de un marco que contiene una clara interrelación con el sistema político (Hallin y Mancini, 2004).

De ahí su nombre: los medios "median" entre los acontecimientos y los públicos que no asisten directamente a estos acontecimientos. Abren "ventas al mundo" a través de los cuales los ciudadanos se conocen a sí mismos y conocen también a los demás y al medio político que les rodea (Tuchman, 1978).

En esta labor de mediación o "mediatización" de la realidad (Strömbäck, 2008) los medios no siempre son neutrales, no seleccionan temas sin más, delegando en las audiencias la construcción autónoma de sus preferencias político-electorales. Como veremos en los datos disponibles, la prensa de referencia tuvo que encuadrar a toda prisa el movimiento de los "indignados", un actor desconocido que irrumpe en el umbral mismo de las elecciones autonómicas y municipales de 2011.

Como suele ser habitual en un sistema mediático polarizado como el español

${ }^{10}$ Para comprobar la presencia o no del 15-M en los medios de comunicación hemos utilizado la prensa de referencia por su capacidad de "contagio" de sus contenidos al resto de familias mediáticas, sobre todo la televisión. Sobre el modelo difusión "en cascada" de los encuadres de la prensa acúdase a González, Rodríguez y Castromil (2010). 
(González, Rodríguez y Castromil, 2010) la labor de encuadre implica incluir una serie de características y dejar fuera muchas otras (Entman, 1993) por criterios no siempre profesionales, sino también políticos.

La polarización de los medios españoles implica que, al menos en el caso de la prensa nacional de referencia que analizamos aquí, los medios se encuentran en clara sintonía con uno de los dos grandes partidos del sistema político español de 2011: el Partido Popular en la oposición o el PSOE del presidente José Luis Rodríguez Zapatero.

Pero este afán por beneficiar al partido o candidato más afín que muestran históricamente El País, El Mundo, ABC o Público puede realizarse de dos formas bien distintas, aunque emparentadas. Se puede hacer o bien ensalzando las virtudes del partido más próximo o bien destacando la inconveniencia y las desventajas de la opción contraria. Se trata, en fin, del uso de la "vía del positivismo" o la "vía del negativismo" a la hora de encuadrar el movimiento del 15-M.

Tal y como veremos en seguida, la prensa tradicionalmente identificada como "de izquierda" o "progresista" (El País y Público) recurrió estratégicamente a la neutralidad ${ }^{11}$ en su cobertura de campaña para dar la "bienvenida" a un movimiento que no se asocia ni positiva ni negativamente con ninguno de los dos grandes partidos. Las cabeceras conservadoras optan, en cambio, por una mayor desconfianza hacia el 15-M y por un empleo más destacado, aunque no generalizado, de la comunicación política negativa ${ }^{12}$. Sin embargo, y en definitiva, el encuadre que los principales periódicos ofrecerán del 15-M está más centrado en la posición ideológica de los partidos más afines que en las reclamaciones e ideas que el nuevo fenómeno social estaba impulsando.

\section{CASO PRÁCTICO}

\subsection{El 15-M y los mass media}

Los movimientos sociales, incluso aquellos que emplean de manera intensiva las tecnologías de la información, siguen necesitando de los medios de comunicación tradicionales para llegar al grueso de la opinión pública. Esto es, la prensa, la radio y, sobre todo, la televisión (Castromil y Resina, 2013).

Esta impresión parece válida para 2011, año en que surge el 15-M (mayo)

11 La codificación del tono en nuestro análisis de contenido se llevó a cabo distinguiendo entre cobertura polarizada y no polarizada. Así, las noticias no polarizadas -también denominadas neutrales- fueron aquellas en las que no se observó relación entre cobertura y defensa de los intereses de una determinada opción política. Sin embargo, en las noticias polarizadas esta relación fue más o menos evidente, aunque la forma de hacerlo tomó dos caminos distintos. A través del "positivismo" (beneficiando a la opción político-partidista afín) o del "negativismo" (atacando al contrario).

12 Tema bien distinto será sopesar el impacto que en los electores tienen este tipo de coberturas negativas: favorecen el alejamiento de la política (Ansolabehere \& Iyengar, 1995) o, por el contrario, refuerzan el sistema democrático (Geer, 2006). 
y se celebran las undécimas elecciones generales de la historia democrática española. Según datos del Centro de Investigaciones Sociológicas (noviembre de $2011^{13}$ ) algo más del $40 \%$ de los ciudadanos no pudo enterarse de la existencia del movimiento de los indignados en el ámbito en el que éste nace, la Red, simplemente porque no la usan.

Pero, además, entre aquellos otros ciudadanos que aseguraron haber utilizado Internet $(58,2 \%)$, el grupo más numeroso $(41,4 \%)$ ha empleado Internet para replicar pautas de exposición mediática tradicionales. Esto es, para acceder a coste reducido a las ediciones on line de medios de comunicación de "toda la vida". En cambio, el seguimiento de la actualidad política a través de modos más interactivos, como las redes sociales o los blogs, ha resultado más bien residual: $17,6 \%$ y $8,9 \%$, respectivamente.

Sin embargo, estos datos, lejos de resultar definitivos, deberán ser contrastados con el paso del tiempo, ya que el principal factor que podría estar operando a favor del uso de las tecnologías de la información es la edad. Ya en noviembre de 2011 se observa que los más jóvenes (18-29 años y 30-39 años) constituyen los grupos de electores más predispuestos a seguir la campaña a través de vías interactivas como los anteriormente señalados blogs y redes sociales. El esquema parece ser el siguiente: conforme avanza la edad decae el empleo de este tipo de "artefactos" comunicativos tal y como muestra el gráfico 1.

GRÁFICO 1: Empleo de tecnologías de la comunicación por edad (2011)

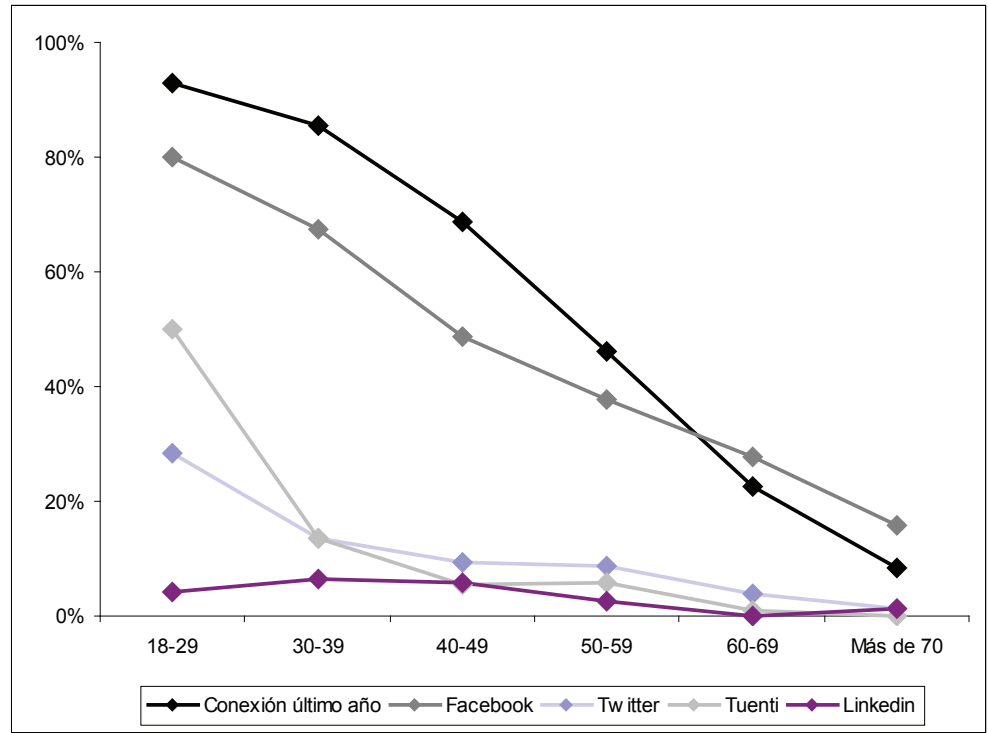

Fuente: CIS (estudio 2920)

${ }^{13}$ Estudio 2920. 
Que Internet ocupe, de momento, un lugar más bien secundario en el menú informativo de la mayoría de los españoles no significa que éstos desconecten de la actualidad política. Más bien revela que continúan nutriéndose, principalmente, de la televisión.

Los datos son reveladores. El 86,5\% de los ciudadanos dice haber seguido la campaña de 2011 a través de la televisión, que sigue siendo hegemónica con mucha diferencia. La radio y la prensa tienen que conformarse con unos índices de seguimiento del $35,8 \%$ y $50,8 \%$, respectivamente.

Esta circunstancia parece indicar que la construcción de la agenda de temas de campaña tiene mucho más que ver con la acción de la televisión, la prensa y la radio que con los medios de nuevo cuño. Al menos de momento.

En este sentido, resulta de interés preguntarse si en el momento en que surge el 15-M el movimiento entró a formar parte o no de los contenidos prioritarios de los medios de comunicación más tradicionales. De entre ellos hemos seleccionado, como mencionamos anteriormente, la prensa nacional de referencia debido a su capacidad de influencia sobre las demás familias de medios.

Llegados a este punto parece lógico plantearse algunas preguntas que el lector atento estará ya masticando: ¿Se ha convertido el 15-M en un tema de campaña? ¿Qué lugar ocupa en la agenda mediática de referencia: prioritario o más bien secundario? ¿Cómo se posiciona: neutralidad o tendenciosidad hacia el movimiento de los "indignados"?

Como se ha descrito, el movimiento de los "indignados" surge en los días previos a las elecciones autonómicas y municipales de 2011 y ofrece su cara más visible en las acampadas de la Puerta del Sol de Madrid y de Plaza Catalunya de Barcelona. Los medios de comunicación, como buena parte de la sociedad española, tienen que vérselas con un fenómeno que eclosiona y toma dimensión estatal poco antes de las elecciones. ¿Quiénes son, qué quieren y a qué actor político benefician/perjudican más?

La construcción de la agenda de temas de las elecciones autonómicas y municipales de 2011 se ve influenciada por el movimiento del 15-M. Sin embargo, se observan también, por comparación a las generales de 2008, por remontarnos al antecedente electoral más próximo, ciertas pautas de continuidad a pesar de la novedad de los "indignados".

En 2008 el principal tema de campaña de El País, El Mundo y ABC fue el terrorismo (41\% de la cobertura) seguido a bastante distancia por cuestiones como la economía $(18,8 \%)$ o la política autonómica $(15,6 \%)$. Los cinco principales temas de agenda se completan con la inmigración y la política social $(12,5 \%$ y $12,1 \%$, respectivamente).

Tres años más tarde (mayo 2011) se repite el esquema: el terrorismo o, más bien, la cuestión de la legalidad o no de las listas de Bildu ${ }^{14}$, ocupa el primer

14 Bildu, formada por Eusko Alkartasuna, Alternatiba y otros grupos independentistas de la denominada izquierda abertzale vasca se constituye en 2011 como coalición electoral para concurrir a las elecciones municipales del País Vasco y Navarra. La formación, en tanto que heredera 
rango de la agenda de campaña con el $45,2 \%$ de la cobertura total de la campaña. De forma similar a 2008, a una cierta distancia se sitúan una serie de temas que también han tenido su importancia en la campaña como son la corrupción $(14,9 \%)$, el $15-\mathrm{M}(13,4 \%)$, el seguimiento de los sondeos $(13,1 \%)$ y la economía $(10,9 \%)$.

Como se puede comprobar en el cuadro 1 la cuestión de los "indignados" permanece entre los cinco temas más importantes de la campaña de las elecciones generales de 2011. Es decir, se convierte en un tema presente en la agenda mediática durante toda una legislatura (2008-2011).

CUADRO $1^{15}$ : Rango de temas (5) en las últimas tres elecciones de ámbito estatal

\begin{tabular}{|l|c|c|c|}
\hline \multicolumn{1}{|c}{} & $\begin{array}{c}\text { Generales } \\
\mathbf{2 0 0 8}\end{array}$ & $\begin{array}{c}\text { Auto y Muni } \\
\mathbf{2 0 1 1}\end{array}$ & $\begin{array}{c}\text { Generales } \\
\mathbf{2 0 1 1}\end{array}$ \\
\hline Terrorismo & 1 & 1 & 2 \\
Economía & 2 & 5 & 1 \\
15-M & - & 3 & 4 \\
Corrupción & - & 2 & 3 \\
Pol. Autonómica & 3 & - & 5 \\
Inmigración & 4 & - & - \\
Pol. Social & 5 & - & - \\
Encuestas & - & 4 & - \\
\hline
\end{tabular}

Fuente: Datos propios

de otras marcas del nacionalismo vasco como Batasuna, protagonizó la polémica de la campaña al ser ilegalizada primero por el Tribunal Supremo (01/05/2011) y vuelta a la legalidad, después, por Tribunal Constitucional (05/05/2011).

${ }^{15}$ El seguimiento de prensa sobre las elecciones generales de 2008 y 2011 y las autonómicas y municipales de 2011 se extendió a la totalidad de la información electoral publicada (primera página, editoriales y páginas interiores) durante el mes y medio previo a la celebración de las elecciones. La codificación fue coordinada por el profesor Antón R. Castromil (UCM) y el método de recogida de la información se basó en la lectura completa de la noticia y la su posterior codificación informática mediante plan estructurado y pre codificado. Ficha técnica del trabajo de campo: Ámbito: Elecciones Generales 2008, Elecciones Autonómicas y Municipales de 2011 y Elecciones Generales de 2011. Universo: Totalidad de la información publicada sobre las elecciones: primera página, editoriales y páginas interiores. Artículos de opinión de colaboradores y cuadernillos especiales excluidos. Tamaño y distribución de la muestra: Generales 2008: 2112 noticias (El País 554 ítems, 26,2\%; El Mundo 778 ítems, 36,8\% y ABC 780 ítems, 36,9\%). Autonómicas y Municipales 2011: 2095 noticias (El País 445 ítems, 21,2\%; El Mundo 603 ítems, 28,8\%; ABC 411 ítems, 19,6\% y Público 636 ítems, 30,4\%) y Generales 2011: 1753 noticias (El País 630 ítems, 35,9\%; El Mundo 593 ítems, 33,8\% y ABC 530 ítems, 30,2\%). Método de recogida de información: Lectura completa de la noticia y codificación informática correspondiente mediante plan estructurado y pre codificado. Seguimiento: Precampaña (30 días aproximadamente); Campaña (15 días) y Post campaña: (3 días). Número de codificadores: 3 Investigador responsable: Antón R. Castromil. Universidad Complutense. 
Pero, junto a la construcción de la agenda de campaña nos interesa destacar también el tono de la cobertura en cada uno de estos temas teniendo en cuenta el periódico concreto que publica la noticia. Este nivel de desagregación de datos resulta imprescindible si lo que se quiere conocer es la posición (encuadre) de la prensa de referencia con respecto al 15-M en tanto que nuevo actor que irrumpe con fuerza en la escena política a partir de mayo de 2011.

Nos centraremos aquí en los datos de las elecciones en las que surge el 15M, las autonómicas y municipales de 2011. De las 156 noticias con temática "indignados" publicadas por la prensa de referencia la mayor parte de ellas, el $36,5 \%$, aparecieron en las páginas de El Mundo. El País y Público ofrecen a sus lectores una volumen de cobertura inferior, aunque muy similar $(22,4 \%$ y $24,4 \%$, respectivamente). El periódico que muestra una menor propensión a cubrir el movimiento es el $A B C$ (16,7\% del total de su cobertura).

Las tendencias más interesantes se detectan cuando tenemos en cuenta la presencia o no de sesgos (tendenciosidad). Es decir, cuando analizamos la relación que se establece entre el medio de comunicación en cuestión, el movimiento del 15-M y su cobertura destinada a la influencia de la opinión pública.

El diario más neutral es, sin duda, El País. El periódico del grupo PRISA no identifica al movimiento de los "indignados" con ninguna tendencia. Su compañero en el espectro ideológico de la izquierda, Público, mantiene también una posición mayoritariamente neutral (84,2\% de su cobertura). Es decir, la prensa "progresista" más próxima a los socialistas aborda el surgimiento del 15-M sin asociarlo negativamente al PP ni positivamente al propio PSOE. Mantiene el tema alejado de la polarización y se limita a informar sobre él.

\begin{tabular}{|c|c|c|c|}
\hline & $\begin{array}{c}\text { Cobertura } \\
\text { sobre el 15-M }\end{array}$ & $\begin{array}{c}\text { Cobertura } \\
\text { neutral }\end{array}$ & $\begin{array}{c}\text { Cobertura } \\
\text { negativa }\end{array}$ \\
\hline El País & $22,4 \%$ & $100 \%$ & - \\
\hline El Mundo & $36,5 \%$ & $71,9 \%$ & $\begin{array}{c}15,8 \% \text { PSOE } \\
12,3 \% \text { Otro }\end{array}$ \\
\hline$A B C$ & $16,7 \%$ & $23,1 \%$ & $\begin{array}{c}30,8 \% \text { PSOE } \\
46,2 \% \text { Otro }\end{array}$ \\
\hline Público & $24,4 \%$ & $84,2 \%$ & $\begin{array}{c}5,3 \% \text { (PP) } \\
2,6 \% \text { (PSOE) }\end{array}$ \\
\hline
\end{tabular}

Fuente: Datos propios

Caso bien distinto lo constituye la prensa conservadora. Se observa en sus páginas una menor tendencia hacia la neutralidad, sobre todo en $A B C(23,1 \%)$, lo que nos ofrece un punto de partida diferente al de El País y Público. 
Los datos parecen indicar que la derecha mediática tiende a considerar al 15-M como un enemigo potencial en su juego de influencias polarizantes: favorecer al PP o, en mayor medida, desprestigiar al PSOE ${ }^{16}$. Tanto El Mundo $(15,8 \%)$ como, sobre todo, $A B C(30,8 \%)$ ofrecen una cobertura negativa al $15-$ M, si bien el diario de Unión Editorial tiende a relacionar ese negativismo con el PSOE en mayor medida que $A B C$.

$A B C$ asigna un sesgo negativo a su cobertura del $15-\mathrm{M}$ pero la dirige no sólo hacia el PSOE del recién nominado candidato Alfredo Pérez Rubalcaba sino, mayoritariamente $(46,2 \%)$, con el propio movimiento. Es decir, presenta a los "indignados" mucho más como un movimiento "malo" en sí mismo, anti sistema, desordenado y contrario a la ley y el orden, antes que como un movimiento oscuramente ligado al PSOE y, sobre todo, contrario a los intereses electorales del Partido Popular.

En resumen, y tal como hemos mencionado más arriba, la imagen que vierten los principales periódicos sobre el 15-M no recoge los intereses de este colectivo, sino que los subsume dentro del marco general de "contienda política partidista" en el que se transforma el panorama político español durante las campañas electorales.

\subsection{El 15-M y su percepción de las redes sociales como una estructura de oportunidad mediática.}

Esta segunda parte del apartado metodológico es de naturaleza cualitativa, en tanto que nuestro propósito es profundizar en las opiniones y actitudes de los activistas del 15-M respecto al uso de las herramientas de comunicación digitales (Twitter, Facebook, etc.). Nos interesa especialmente enfatizar el hecho de que dichos activistas contaban con una estrategia clara y consciente de lucha por el control de la información difundida sobre el movimiento y que, para dicha pugna, las redes sociales se transformaron en el canal más propicio. El 15-M se moverá durante este periodo en la ambivalente posición de desconfiar de los medios y, al mismo tiempo, necesitarlos para alcanzar a poblaciones más amplias.

El colectivo seleccionado, al que hemos llamado genéricamente 'activistas del 15-M', estaría formado por aquéllas personas que, por su protagonismo en las redes sociales usadas por el 15-M o porque directamente se encargaron de su coordinación, tuvieron directa o indirectamente un papel fundamental en la política de comunicación digital del movimiento.

Las variables de segmentación utilizadas en la selección de los activistas con los que se ha trabajado son: género, edad y haber formado parte con anterioridad de organizaciones o movimientos sociales. La técnica de recogida de la información ha consistido en entrevistas en profundidad semi-estructuradas. Se realizaron un total de 12 entrevistas y, para este trabajo, hemos extraído un

16 Sobre el uso extensivo del negativismo como forma de favorecer al candidato o partido cercano en la prensa de referencia española acúdase a Castromil (2012). 
conjunto de testimonios en los que se recogen las opiniones sobre los siguientes temas: papel de internet desde el inicio del movimiento 15-M y uso de las redes sociales como contrapoder mediático.

Para los entrevistados, Internet está en la propia génesis del movimiento 15-M. Según los activistas entrevistados, Internet es un espacio de referencia para el desarrollo de Internet ya que se transforma en un vehículo clave para la construcción del marco de diagnóstico del movimiento sobre la realidad política y social española.

El 15-M como tal fue convocado por un grupo de gente que se comunicaba a través de Internet. Por eso se pudo hacer en ciudades de todo el estado a la vez. También la lucha contra la Ley inde y el movimiento no les votes creo que fueron unos de las corrientes a través de las que se canalizó el descontento (Entrevistada 3, Mujer, Madrid, activista del 15-M).

También en consonancia con los estudios clásicos sobre movimientos sociales e Internet, encontramos testimonios de los participantes según los cuales este medio será de especial importancia para la gestión y organización de la acción colectiva. En este sentido, vemos cómo Internet, en general, y las redes sociales, en particular, se transforman en canalizadores primero del malestar y "el cabreo" para, posteriormente, convertirse en el "caldo de cultivo" para la acción directa.

Sin embargo, lo más relevante para nuestro objeto de estudio es que, desde el principio, existe una percepción de que 1) la información es un estímulo básico para la movilización, 2) que los mass media gracias a sus intereses corporativos no harán llegar a la población general los intereses y preocupaciones emergentes entre los ciudadanos y 3) que Internet era el medio que permitía romper la relación de poder asimétrica que establecen los mass media en relación a la gestión interesada de la información.

Yo recuerdo una época a partir de marzo así que yo cada día recibía en mi mail o vía twitter o lo que sea noticas de este tipo en las que notabas que la gente estaba cabreada y que había una predisposición a la movilización y que había más gente hablando de política en Internet y compartiendo información sobre temas políticos que llamaban a la movilización. Ese caldo de cultivo para salir a las plazas no hubiera sido posible sin Internet. Por que los medios masivos o corporativos no difunden esta información o no le dan espacio (entrevista 2, mujer, Madrid, activista del 15-M).

Para algunos de los activistas, las nuevas herramientas tecnológicas suponen una ruptura en la mediación entre los medios y los ciudadanos. El papel de tamiz que tradicionalmente han tenido los medios filtrando la realidad, es modificado cuando los ciudadanos, gracias a sus testimonios directos, ofrecen información. Así, éstos se transforman en periodistas amateur (Sampedro, 2014). En el ejemplo que mostramos aquí, el entrevistado nos habla sobre la publicidad que tienen, gracias a las cámaras que los ciudadanos portan en sus teléfonos móviles, los casos de abuso de la fuerza por parte de la policía. La cuestión, nos dice, no 
es que exista más violencia, sino que, gracias a la independencia informativa de los ciudadanos, ahora toda acción violenta se hace accesible.

La otra cuestión clave es que la libertad en la gestión de la información permite la construcción independiente y crítica de la realidad. Los ciudadanos que ven "lo que pasa" a través de los ojos de otros ciudadanos, despiertan y ven con ojos críticos la realidad. Sin embargo, es importante destacar cómo esta posibilidad es explicada, nuevamente, mostrando el antagonismo con los efectos de consumir información de los mass media. De la misma forma en que la información desintermediada nos libera, nos hace ver cómo la información mediatizada sirve a los intereses parciales de partidos y empresas. Esta explicación recuerda claramente a la famosa proclama del movimiento "dormíamos y despertamos".

Hay una frase bastante interesante que se dijo durante este verano cuando hubo todos esos episodios de violencia policial y de gente, se decía que no es que los policías pegaran más ahora, sino que los móviles son mejores. El hecho de grabar en vivo todos estos episodios que fueron sucediendo fue clave para despertar a gente a un pensamiento crítico de la realidad política que vivimos y de cómo hay que desconfiar de los medios de comunicación de masas que solo hacen servir a los intereses para los que trabajan (entrevistado 2, hombre, Madrid, activista del 15-M).

Desde nuestro punto de vista, parece claro que, cuando el movimiento pasa de su periodo de gestación a su periodo de mayor actividad, el discurso sobre los intereses partidistas de los mass media y de la necesidad de generar espacios de información sin la mediación de estas organizaciones toma una forma mucho más clara y objetiva: se transforma en una estrategia consciente del movimiento.

En esta estrategia, contra-informar, es decir, ofrecer información que pone en cuestión la versión de la realidad ofrecida por los mass media y, por otro lado, generar información independiente cuyo objetivo es ofrecer una representación crítica de la situación social y política de España, se transforman en prioridades para los activistas consultados.

Durante la acampada el objetivo [del twitter del 15-M] era movilizar a la gente en torno a la acampada, en un principio también fue recabar cosas que faltaban, aunque esto se dejó de hacer relativamente pronto, y en todo caso un medio de comunicación en el cual por un lado se contra-informa respecto a lo que cuentan los medios clásicos de información y por otro lado se hacen llamamientos y se difunde información crítica (entrevistado 1, hombre, Madrid, activista del 15-M).

Esta estrategia alcanza, siempre en palabras de los activistas, su objetivo cuando el movimiento 15-M logra posicionar sus redes sociales (Twitter principalmente) como una fuente de información, no solo para sus activistas, sino también para los propios medios. El testimonio que reproducimos a continuación es especialmente relevante en este sentido ya que narra cómo la información que producen algunos activistas del movimiento es utilizada 
directamente y sin mediación del productor de la noticia.

Desde nuestro punto de vista este logro es esencial ya que en él se muestra cómo el movimiento 15-M alcanza un importante control sobre la información. Este control es triple. Por una parte, se controla y verifica la información que ofrecen los medios (contra-información), por otra de ofrece información alternativa para los simpatizantes y activistas (crítica) y, por último, los propios activistas se transforman los productores de las noticias para los medios. Sin duda, desde el punto de vista de este entrevistado, el movimiento ha sido capaz de ejercer un importante control sobre la información minimizando la mediación mediática.

Hubo momentos, a mi personalmente me ha sucedido, twitear información de lo que estaba sucediendo en la calle y casi instantáneamente ver en la televisión un programa de debate o de lo que sea, en los subtítulos que suelen pasar, exactamente mis palabras como fuente solvente de información crítica (entrevistado 1, hombre, Madrid, activista del 15-M).

Como colofón, queríamos compartir este testimonio que resume el logro mediático del movimiento 15-M y que, desde nuestro punto de vista, justifica una artículo que, como este, pone sobre la mesa la pugna entre el 15-M, y su uso de Internet, y los medios de comunicación tradicionales en España.

Internet se transforma en nuestro medio para poner en cuestión lo que no decían los medios. A lo largo de todo este proceso, nuestro Twitter era el más seguido por encima de líderes de opinión, políticos, partidos políticos y demás.. (entrevistado 1, hombre, Madrid, activista del 15-M).

\section{CASO CONCLUSIÓN}

Este trabajo analiza el uso político de Internet por parte del movimiento social 15-M, tanto las posibilidades que este ofrece como las estrategias utilizadas a partir de las redes sociales. También se analiza, el papel de los medios tradicionales, en concreto la prensa escrita, como medio de información y de estructuración de la opinión pública sobre este nuevo fenómeno político y sus implicaciones sociales.

La justificación e importancia de este análisis reside en que la irrupción del 15-M en el contexto de la gran crisis financiera y económica iniciada en 2008 supone la emergencia de un nuevo campo discursivo que comenzará a disputar el orden simbólico instituido en la sociedad española y el propio orden político. La crisis de los esquemas de sentido que habían servido para ordenar el campo político nos señala el inicio de la crisis de legitimidad del sistema político y la ruptura iniciada por el movimiento 15-M en el contexto de una sociedad mediática y de la información (Castells, 2010).

En este trabajo sostenemos que los dispositivos de comunicación digital, en 
especial internet, son un espacio central de intervención política que permitió al movimiento $15-\mathrm{M}$, además de generar y difundir un discurso (marco), construirse como actor político más allá de los marcos convencionales de sentido que son articulados y re-producidos por los medios de comunicación tradicionales. Como apuntamos, la estructura de oportunidades mediática-digital ha abierto una oportunidad histórica para que las "multitudes" "colaborativas" (Hardt y Negri, 2005) puedan convertirse en agencias de persuasión y significación colectiva (Laraña, 1999) que compitan con las estructuras de producción ideológica ya constituidas en la sociedad, de tal forma que, puedan hacer resonar los marcos de interpretación de forma extensiva.

Como paso previo al análisis empírico se han revisado las interpretaciones existentes sobre la relación entre participación política y herramientas digitales, en concreto las tesis de la normalización (tránsito "natural" de la participación off-line a la on-line) igualación (extensión pluralista de la democracia) e desintermediación (nuevas formas de relación política con el apoyo de nuevas tecnologías). En este trabajo se adopta de manera general este último enfoque ya que nos ofrece conceptos teóricos útiles para comprender y analizar la lógica y el uso político que le han otorgado los promotores y activistas, en tanto que productores de ideología, del $15-\mathrm{M}$. Estos perciben y significan a las redes digitales como dispositivos tecnológicos de poder en la medida en que les permite romper con una relación a-simétrica de partida con los medios tradicionales y ofrecer una representación alternativa de la realidad fuera de los intereses corporativos y políticos de la industria mediática. Esta interpretación estaría en la línea de autores que, como Benkler (2006), plantean que las tecnologías digitales permiten la ruptura con las formas de producción cultural industriales.

La representación que los activistas realizan de los medios convencionales es profundamente negativa, ya que perciben a estos como instrumentos de distorsión y desmovilización política. Sus presupuestos de partida les conducen desde un inicio a plantear una estrategia (visión instrumentalista) consciente y planificada basada en el uso exclusivo de las redes digitales y multimodales. Cabe preguntarse si esta elección tiene tan sólo un carácter intelectual o si la falta de oportunidad de acceso a los grandes medios tradicionales les lleva a privilegiar y legitimar internet como un dispositivo sin "corromper".

Sin duda, el uso político de las redes digitales, con alta penetración en diversas capas sociales, brindó al movimiento de indignados una oportunidad estructural para poder generar un alternativa política al orden social instituido, pero no olvidemos que no se presentó como pura exterioridad al sistema, sino que lo hizo dentro del sentido común de época (tal como señala Gramsci, 1976). Aunque la estrategia del movimiento se desarrolló a través de los medios no convencionales, sus activistas no renunciaron a re-significar los significantes que operan como anclajes de sentido en nuestra sistema político (Justicia, Democracia, entre otros) primando una estrategia discursiva desde el interior del sistema simbólico y evitando todo atisbo de marginalidad o radicalidad.

$\mathrm{El}$ análisis empírico realizado de la prensa convencional, tanto progresista 
como conservadora (que clasificamos conscientes de que ello supone una suerte de esquematismo) nos demuestra que: los medios tradicionales de comunicación jugaron un papel clave en la interpretación y orientación de la opinión pública respecto del 15-M. Un papel al que no renunciaron arrojando sobre la opinión pública una visión polarizada del nuevo movimiento social.

Es por ello que la riqueza y profundidad de este artículo se haya en gran medida en el contra-peso analítico que introducimos desde la perspectiva de los medios de comunicación tradicionales, que alimenta una nervadura y tensión intelectual que enriquece y complejiza el fenómeno que estudiamos. La preeminencia que otorgan a los dispositivos de comunicación digitales los miembros del movimiento de indignados es puesta en cuestión por la vigencia hegemónica de los medios de comunicación tradicionales a la hora de construir la imagen pública del 15-M. De hecho en la construcción de la agenda política, en el encuadre de los temas y en los impactos buscados, el peso de los medios tradicionales, con la TV a la cabeza, juegan un papel dominante, siendo las redes sociales un medio muy activo, creciente y de enorme futuro en el desarrollo de nuevas formas de participación política pero aún limitadas por el peso de las formas tradicionales.

Tal como pone de manifiesto el análisis cualitativo, el uso de las redes digitales con su capacidad de crear información desintermediada o no mediada por los medios tradicionales, así como la capacidad de crear estrategias de contra-información y de ofrecer una información alternativa, depende tanto de la existencia de los nuevos medios digitales como también, y sobre todo, de la capacidad de movilización de un segmento de edad joven que es capaz de acceder y utilizar dichos medios en una nueva dirección que les hace ser creadores de nuevas formas y contenidos de la información política.

En base a todo lo anterior se pueden destacar cuatro ideas centrales desde la teoría de los mass media. En primer lugar, los movimientos sociales dependen aún de los medios de comunicación tradicionales para poder llegar al conjunto de la sociedad. En segundo lugar, esto se explica en parte por la existencia de una brecha digital que tiene fundamentalmente una lectura de carácter educativo y generacional (Robles y Torres, 2012). En tercer lugar, una parte numerosa de ciudadanos han intervenido en internet replicando los marcos discursivos generados en los mass media. Y por último, la construcción de la imagen pública que los medios tradicionales han realizado del 15-M ha tenido objetivos políticos diferenciados según nos situemos en la esfera progresista (con diferentes niveles de neutralidad) o conservadora (sesgo tendencioso).

El análisis realizado nos muestra que una parte amplia de las personas que intervienen políticamente en internet replican o reproducen en gran medida "las pautas de exposición mediática." Por ello, aunque asumido aquí como enfoque general, cabe limitar parcialmente la tesis de la des-intermediación, es decir, vivimos en una sociedad mediada y mediatizada en la que los medios tradicionales tiene aún la posibilidad de seguir reproduciendo los saberes y creencias que legitiman el/los discurso/s hegemónico/s (que condicionan la percepción de la realidad social y política), dando fuerza a la episteme epocal 
dominante (en palabras de Foucault, 1968). Por este motivo, si bien las redes digitales permiten desplegar una estrategia basada en la des-intermediación, ésta aún no es total, siempre es precaria y contingente, limitada por la influencia de los medios tradicionales en las formas de pensar y enmarcar de las personas, así como por el propio orden discursivo de la época.

Por ello, es necesario limitar un cierto optimismo tecnológico ante una realidad dominada aún por los medios tradicionales, si bien indicamos que la tendencia será a un papel preponderante de los nuevos medios ante el avance inexorable del cambio generacional. En este sentido, pensar la estrategia e impacto comunicativo del 15-M no puede limitarse a la dimensión digital, sino que debe pensarse dentro de un ecosistema mediático y digital complejo que se constituye como un campo con distintas posiciones de relación y poder en el que los distintos agentes deben inscribir sus discursos teniendo en cuenta las contradicciones y alcances posibles.

El alcance que logra internet es aún limitado para hacer extensivo los mensajes de cambio social, lo que obliga a los movimientos sociales a reflexionar sobre la necesidad de incorporarse a los medios tradicionales si quieren constituirse como agentes de cambio real.

Para finalizar, quisiéramos apuntar que la disputa por la atribución de sentido emprendida por el 15-M no habría sido posible sin la capacidad de difusión que posibilita estructuralmente los dispositivos de comunicación en las sociedades de la información.

\section{BIBLIOGRAFÍA}

ANSOLABEHERE, S. \& IYENGAR, S. (1995): Going Negative. How Political Advertisements Shrink \& Polarize the Electorate. New York. The Free Press.

ANTÓN, A. (2012): "Movimiento 15-M: expresión colectiva de una ciudadanía indignada", trabajo presentado en la Jornadas de Sociología de la Asociación Madrileña de Sociología, Universidad Complutense de Madrid, marzo.

BECK, U. (1992): Risk Society. Towards a New Modernity, London, Sage.

BENKLER, Y. (2006): The Wealth of Networks: How Social Production Transforms Markets and Freedom, New Haven and London, Yale University Press.

BENNETT, L (2003): Communicating Global Activism: Strengths and Vulnerabilities of Networked Politics, Information, Communication and Society, 6 (2), pp. 143-168.

BENNETT, L. \& SEGERBERG, A. (2013): The Logic of Connective Action: Digital Media and the Personalization of Contentious Politics. Cambridge, Cambridge University Press.

BIMBER, B. (2001): Information and Political Engagement in America: The Search for Effects of Information Technology at the Individual Level, Political Research Quarterly, 54 (1), pp. 53-67.

CANDÓN, J. (2013): “Toma la calle, toma las redes: El movimiento 15M en Internet", Atrapasueños Soc. Coop. And.

CASTELLS, M. (2010): Comunicación y poder. Madrid, Alianza.

(2012): Networks of outrage and hope. Social movements in the Internet Age, Cambridge, Polity Press. 
CASTROMIL, A. R. (2012): "Negativismo mediático y campaña electoral en las elecciones generales de 2008". REIS, 139, pp. 163-174.

CASTROMIL, A. R. y RESINA, J. (2013): "La prensa tradicional en la España del $15 \mathrm{M}$ " en Actores y demandas en España. Análisis de un inicio de siglo convulso. Madrid. Catarata, pp. 107-129.

CHADWICK A. (2006) Internet Politics: States, Citizens, and New Communication Technologies, Oxford, Oxford University Press.

CHADWICK, A. \& HOWARD P.N. (2009): The Routledge Handbook of Internet and Politics, New York, Routledge.

DE MARCO, S., MOLINA, O., ROBLES, JM. (2012): "Participación política digital y brecha digital en España. Un estudio de las desigualdades digitales", Arbor: ciencia, pensamiento y cultura, 756, pp. 795-810

DÍEZ, R. (2013): "An approach to the Spanish 15M and the transnational networks of 'indignados': ideology, identity \& method", presentado en el Workshop 'Transnational public participation \& social movement activism', Lisbon, 14-15 de Noviembre.

(2014): "Does the Spanish 15M have an ideology? Issues of method and measurement", en Athenea Digital - Revista de pensamiento e investigación social, 4(3), pp. 199-217, doi: 10.5565/rev/athenea.1252.

(2015): "Las bases sociales de la indignación: una perspectiva agregada sobre los factores asociados a la participación ciudadana en el movimiento 15M", Sistema: Revista de ciencias sociales, 238, pp. 41-84.

DYLKO, I.B., BEAM, M.A., LANDREVILLE, K.D. \& GEIDNER, N. (2012): Filtering 2008 US Presidential Election News on YouTube by Elites and Nonelites: An Examination of the Democratizing Potential of the Internet, New Media and Society, 145 , pp. 832-849.

ELOLA, J. (2011): “\#SPANISHREVOLUTION. El 15-M sacude el sistema”, Diario El Pais, 22 may. 2011, http://elpais.com/diario/2011/05/22/ domingo/1306036353 850215.html

ENTMAN, R. (1993): "Framing: Toward clarification of a Fractured Paradigm". Journal of Communication, Vol. 43, 4, pp. 51-58.

FERNÁNDEZ-SAVATER, A. (2011): "Una revolución de personas", Público.es, 20 sep. 2011,

http://blogs.publico.es/fueradelugar/920/una-revolucion-de-personas

(2012): “CCómo se organiza un clima?, en Público.es, 09 ene. 2012, http://blogs. publico.es/fueradelugar/1438/\%C2\%BFcomo-se-organiza-un-clima

(2014): "La política y la Nada: España en la crisis", eldiario.es, 10 ene. 2014, http://www.eldiario.es/interferencias/politica-Espana-crisis 6 216688353.html

FOMINAYA, C. (2014): "Debunking Spontaneity: Spain's 15-M/Indignados as Autonomous Movement", Social Movement Studies, September 2014, doi: $10.1080 ! 14742837.2014 .945075$.

FOUCAULT, M. (1968): Las palabras y las cosas. Una arqueología de las ciencias humanas, Siglo Veintiuno.

GEER, J. G. (2006): In Defense of Negativity. Attack Ads in Presidential Campaigns. Chicago, The University of Chicago Press.

GÓNZALEZ, J. J; RODRÍGUEZ, R. y CASTROMIL, A.R (2012): "Dilemas metodológicos en un estudio longitudinal de la influencia de los medios en el voto" en Trucos del oficio de investigador, Casos prácticos de investigación social, Barcelona. Gedisa. 
GÓNZALEZ, J. J; RODRÍGUEZ, R. y CASTROMIL, A.R (2010): “A Case of Polarized Pluralism in a Mediterranean Country. The Media and Politics in Spain", Global Media Journal, 5 (1/2).

GRAMSCI, A. (1976): Selection from the prison notebooks, Lawrence \& Wishart.

HALLIN, D. C. \& MANCINI, P. (2004): Comparing Media Systems. Three Models of Media and Politics, New York. Cambridge University Press.

HARDT, M. y NEGRI, A. (2005): Imperio, Paidós.

JOHNSTON, H., LARAÑA E. y GUSFIELD, J. (1994): "Identidades, ideologías y vida cotidiana en los nuevos movimientos sociales", en LARAÑA, E. y GUSFIELD, J. (eds.), Los nuevos movimientos sociales; de la ideología a la identidad, Madrid, CIS.

KLANDERMANS, B. (1984): "Mobilization and participation: socialpsychological expansions of resource mobilization theory", American Sociological Review, 49.

LARAÑA E. (1999): La construcción de los movimientos sociales, Madrid, Alianza. (2007): "Asociación y modernización social. Las organizaciones voluntarias en España", en Lo que hacen los sociólogos, libro homenaje a Carlos Moya, CIS, Madrid, pp. 735-754.

(2009): "Is Still Spain a Statist Society? A Research Perspective on Organizations, Reflexivity and Collective Action", Institute for the Study of Social Change, UC, Berkley, http://escholarship.org/uc/item/1034d4wj

LARAÑA E. y DÍEZ, R. (2010): "Las grandes manifestaciones en la prensa y el poder de persuasión de las organizaciones cívicas. Metodología de investigación y marcos de movilización", Informe Técnico para el CIS, enero 2010.

(2012): Las raíces del movimiento 15-M. Orden social e indignación moral", Revista Española del Tercer Sector, 20, pp. 105-144.

(2013): "Las organizaciones reflexivas y el surgimiento de la sociedad civil en España”, presentado en el XI Congreso Español de Sociología (Ponencia del Grupo Movimientos Sociales, Acción Colectiva y Cambio Social), Madrid, UCM, julio.

MACINTOSH, A. (2006): E-Participating in Policy-Making: The Research and the Challenges, en CUNNINGHAM, P. \& CUNNINGHAM M. (eds.), Exploiting the Knowledge Economy: Issues, Applications, Case Studies, Amsterdam, IOS Press.

MAECKELBERGH, M. (2012): "Horitzontal Democracy Now: From AlterGlobalization to Occupation", Interface: a Journal for and about Social Movements, 4 (1), pp. 207-234.

McCOMBS, M. y SHAW, D. (1972): "The Agenda Setting Function of the Mass Media”, Public Opinion Quarterly, 36, pp. 176-187.

McCOMBS, M et al. (1998): “Cantidate's Images in Spanish Elections: Second-Level Agenda-Setting effects", Journalism \& Mass Communication Quarterly, 74 (4), pp. 703-717.

MELUCCI, A. (1989): Nomads of the Present, Philadelphia, Temple University Press. (1996): Challenging Codes. Collective Action in the information age. Cambridge, Cambridge University Press.

PERUGORRÍA I. y TEJERINA, B. (2013): "Politics of the encounter: Cognition, emotions, and networks in the Spanish 15M", Current Sociology, 61 (4), pp. 424442.

ROBLES, J. M. y GANUZA, E. (2011): “Internet y Deliberación. Dos ideas para comprender cómo afrontan los Indignados la participación política", Sociedad y Utopía, 3, pp. 243-262.

ROBLES, J. M. y TORRES-ALBERO, C. (2012): "Digital Divide and the Information and Communication Society in Spain”, Sociology and Space, Vol. 194 (3): 291-307. 
ROBLES, J. M (2015). E-gobierno y Ciberpolítica, Informe de Situación Social 2015, Madrid, Centro de Investigaciones Sociológicas.

RODRÍGUEZ, R. y CASTROMIL, A. R. (2010): "La circulación social de los encuadres periodísticos en tiempo de campaña electoral: Transmisión, influencia y atribución de responsabilidad", ZER, Vol. 15, 29, pp. 193-212.

SAMPEDRO V. (2014): El cuarto poder en red. Por un periodismo (de código libre) libre, Madrid, Icaria.

SAMPEDRO V. \& LOBERA, J. (2014): “The Spanish 15-M Movement: a consensual dissent?", Journal of Spanish Cultural Studies, 15, Issue 1-2, pp. 61-80, doi: $10.1080 / 14636204.2014 .938466$

STRÖMB̈̈CK, J. (2008): "Four Phases of Mediatization: An Analysis of the Mediatization", International Journal of Press/Politics, 13, pp. 228-246.

TORET, J. (2012): "Una mirada tecnopolítica sobre los primeros días del \#15M", en Alcazan, ArnauMonty, Axebra, Quodlibetat, Simona Levi, SuNotissima, TakeTheSquare y Toret (eds.) Tecnopolítica, internet y r-evoluciones, Barcelona, Icaria.

TUCHMAN, G. (1978): Making News. A Study of Construction of Reality, New York, The Free Press.

VAN DIJK, J. (2000): Models of Democracy and Concepts of Communication, en HACKER K.L. \& VAN DIJK, J. (eds.), Digital Democracy: Issues of Theory \& Practice, Thousand Oaks, Sage Publications.

WARD, S. \& GIBSON, R. (2009): European Political Organizations and the Internet: Mobilization, Participation, and Change, en CHADWICK, A. \& HOWARD, P.N. (eds.), The Routledge Handbook of Internet Politics, New York, Routledge.

WILLIAMS, C.B. \& GULATI, J. (2013): Social Networks in Political Campaigns: Facebook and the Congressional Elections of 2006 and 2008, New Media and Society, 151, pp. 52-71.

WRING, D. \& HORROCKS, I. (2001): The Transformation of Political Parties, en AXFORD, B. \& HUGGINS, R. (eds.), New media and Politics, Longon, Sage. 
